\title{
Non fermentative gram negative bacilli as nosocomial pathogens: Identification and antibiotic sensitivity in clinical samples of indoor patients
}

\author{
Sharma D, ${ }^{1}$ Vyas $N,{ }^{1}$ Sinha $P,{ }^{1}$ Mathur $A^{2 *}$ \\ ${ }^{1}$ Department of Microbiology, SMS Medical College, Jaipur, India, \\ ${ }^{2}$ Department of Microbiology, Gulf Medical University, Ajman, United Arab Emirates.
}

*Corresponding Author:

Dr. Anuj Mathur, Assistant Professor

Department of Microbiology,

Gulf Medical University,

Ajman, United Arab Emirates

Email id: dranujmathur@gmail.com

\begin{abstract}
Background: Non fermenting gram negative bacilli (NFGNB) are usually considered to be non pathogenic commensals of little significance, however, data suggest that they are remarkable because of antimicrobial resistance; propensity to cause outbreaks and complex epidemiology. This study was conducted to find out the prevalence and sensitivity pattern of NFGNB from various clinical samples.
\end{abstract}

Methods: A total of 519 samples were received from patients suspected of having hospital acquired infections (HAI), admitted in Sawai Man Singh Hospital, Jaipur, India. Organisms grown on culture were subjected to phenotypic identification along with antimicrobial sensitivity testing by Kirby Bauer's disc diffusion method.

Results: Among 366 culture positive samples, 94 (25.6\%) NFGNB were isolated. Maximum pevalence prevalence was found in burn ward i.e. 76.7 $\%$ followed by surgical wards $71.0 \%$, surgical ICUs $69.6 \%$ and medical ICUs $68.0 \%$. Highest yield was found in pus/wound samples $(95.5 \%)$ while it was only $23.7 \%$ in blood samples. Pseudomonas aeruginosa was the most common isolate (48.9\%), followed by Acinetobacter baumannii (33\%), Stenotrophomonas maltophilia (13.8\%) and Burkholderia cepacia complex (4.3\%). P.aeruginosa and A. baumanii strains were most sensitive to Meropenem. S.maltophilia showed maximum sensitivity with ticarcillin/ clavulanic acid and B. cepacia complex with Piperacillin/ Tazobactam.

Conclusion: Isolation of NFGNB and their antibiotic susceptibility pattern should be regarded with all seriousness in clinical practice and epidemiology because they are emerging nosocomial pathogens and by being resistant to multiple antibiotics, their prevalence not only limits the treatment options but also act as a reservoir of drug resistance genes.

Keywords: Hospital acquired infections; multi drug resistance; non fermenting gram negative bacilli

\section{Background}

Nonfermenting gram negative bacilli (NFGNB) are although considered commensals or contaminants; their pathogenic potential has been well-established by their frequent isolation from clinical samples and their association with clinical disease. The outbreaks of nosocomial infections, emerging antimicrobial resistance and epidemiological complexity have made NFGNB the remarkable organisms. ${ }^{1}$ Very few laboratories in India identify these NFGNB other than Pseudomonas routinely. This may be because they require a set of unique biochemical reactions for identification which 
is time as well as resource-consuming and other reason is that the importance of their species identification is still undermined. ${ }^{1}$

NFGNB are resistant to multiple classes of antibiotics and resistance is due to the mobile genetic elements such as plasmids and transposons that may be transferred between and across different bacterial species with relative ease. ${ }^{2}$ This emphasizes the importance of inclusion of tests for their isolation and identification routinely, which can throw more light on the prevalence and pathogenic role of these organisms. ${ }^{3}$

The importance of the study of sensitivity pattern, as emphasized by various international authorities is that every hospital should have its individual antibiotic sensitivity pattern since the standard antibiotic sensitivity pattern may not hold true for every area. ${ }^{4}$

In view of these facts current study was undertaken to find out the prevalence as well as the antibiotic sensitivity pattern of NFGNB (Pseudomonas and others) in various clinical samples.

\section{Methods}

Present study was conducted in the Department of Microbiology \& Immunology, Sawai Man Singh (SMS) Medical College, Jaipur, India over a time period from August 2009 to May 2010. A total number of 519 specimens were received from patients of different age groups admitted in ICUs (medical and surgical), surgical and burn wards of SMS Hospital, Jaipur, suspected of suffering from nosocomial infections. Nosocomial infection was defined as per Centre for diseasecontrol definition. ${ }^{5}$

Organisms grown from all the specimens like blood, body fluids, tracheal aspirates, pus, swabs, sputum and urine were subjected to phenotypic identification along with antimicrobial sensitivity testing.

\section{Sample processing}

Specimens were cultured on blood agar (BA), MacConkey agar (MCA) and Thioglycolate broth (TG) and gram staining was done of all the samples. Culture plates were observed after $18-24 \mathrm{hrs}$ of aerobic incubation at $37^{\circ} \mathrm{C} \&$ further, if felt necessary. If multiple organisms were grown on culture plate, colonies were isolated further by subculture. Blood specimens were inoculated in Brain heart infusion broth and then subcultured on BA and MCA after 48 hours and 7 days of incubation. From the culture plates, again the smears were made. Battery of biochemical reactions including oxidase test by Kovac's method, triple sugar iron (TSI) test, sulphideindole motility (SIM) test, Hugh \& Leifson oxidation fermentation dextrose test, gelatin hydrolysis test, decarboxylase test along with motility testing and testing growth at $42^{\circ} \mathrm{C} \& 44^{\circ} \mathrm{C}$ for differentiation of non fermenters ${ }^{6}$ were done for further identification of the organism using positive and negative controls for each test.

Antibiotic sensitivity testing was done by Kirby Bauer method as per CLSI guidelines. ${ }^{7}$ Following antibiotics discs (Himedia) were used: Amikacin, Aztreonam, Ceftazidime, Ciprofloxacin, Gentamicin, Piperacillin, PiperacillinTazobactem, Tobramycin, Meropenem and TicaricillinClavulinic acid.

\section{Results}

A total of $70.5 \%(366 / 519)$ samples were found to be culture positive, out of which $25.6 \%$ (94/366) were non fermenters (NF). Male : female ratio among the patients having infections with NFs was found to be 1:1. With age, there seemed to be an increasing trend of infections with NFs.

Maximum percentage of NFs was from burn ward i.e. 76.7 $\%$ (33/43) followed by surgical wards $71.0 \%(174 / 245)$, surgical ICUs $69.6 \%$ (78/112) and medical ICUs $68.0 \%$ (81/119).

Sample wise culture positivity is shown in table 1. Maximum positivity was found among pus/wound samples (95.5\%) while only $23.7 \%$ of blood samples collected were found to be culture positive.

Table 1: Prevalence of nosocomial infections in different clinical samples

\begin{tabular}{|c|c|c|c|c|c|}
\hline \multirow{2}{*}{ Specimen } & \multirow{2}{*}{$\begin{array}{c}\text { Total } \\
\text { no. }\end{array}$} & \multicolumn{2}{|c|}{$\begin{array}{l}\text { Culture } \\
\text { positive }\end{array}$} & \multicolumn{2}{|c|}{ Sterile } \\
\hline & & $\begin{array}{c}\text { Total } \\
\text { no. }\end{array}$ & $\%$ & $\begin{array}{c}\text { Total } \\
\text { no. }\end{array}$ & $\%$ \\
\hline Respiratory specimens & 165 & 145 & 87.9 & 20 & 12.1 \\
\hline Pus/ Wound swab & 110 & 105 & 95.5 & 05 & 4.5 \\
\hline Urine & 153 & 87 & 56.9 & 66 & 43.1 \\
\hline Blood & 72 & 17 & 23.7 & 55 & 76.3 \\
\hline Others* & 19 & 13 & 68.4 & 06 & 31.6 \\
\hline Total & 519 & 366 & 70.52 & 153 & 29.48 \\
\hline
\end{tabular}

*Various samples as tissue,chest drain, ET tip, body fluids etc. were included together in other samples category.

Gram negative bacilli (GNB) were the most commonly isolated organisms $(72.4 \%)$, out of which $35.5 \%$ were NFs. Rest of them were either gram positive cocci $(16.7 \%)$ or candida (10.9\%).

Sample wise distribution pattern of NFs is depicted in table 2. Respiratory specimens was the most common source. Pseudomonas aeruginosa was the most commonly isolated NF i.e. $48.9 \%$ (46/94), followed by $33 \%$ (31/94) Acinetobacter baumannii, 13.8\% (13/9) Stenotrophomonas maltophilia and 4.3\% (04/94) Burkholderia cepacia 
complex.

Table 2: Distribution of nonfermenters in various clinical samples

\begin{tabular}{lccccc}
\hline \multicolumn{1}{c}{ Specimen } & $\begin{array}{c}\text { P.aerugi- } \\
\text { nosa } \\
\text { No. }(\%)\end{array}$ & $\begin{array}{c}\text { A.bau- } \\
\text { mannii } \\
\text { No. }(\%)\end{array}$ & $\begin{array}{c}\text { S.malto- } \\
\text { philia No. } \\
(\%)\end{array}$ & $\begin{array}{c}\text { B. cepacia } \\
\text { complex } \\
\text { No. }(\%)\end{array}$ & Total \\
\hline $\begin{array}{l}\text { Respiratory } \\
\text { specimens }\end{array}$ & $21(47.8)$ & $14(31.8)$ & $07(15.9)$ & $02(4.5)$ & 44 \\
$\begin{array}{l}\text { Pus/Wound } \\
\text { swab }\end{array}$ & $16(53.3)$ & $09(30)$ & $04(13.4)$ & $01(3.3)$ & 30 \\
Urine & $06(50)$ & $03(25)$ & $02(16.7)$ & $01(8.3)$ & 12 \\
Blood & $01(50)$ & $01(50)$ & Nil & Nil & 02 \\
Others & $02(33.3)$ & $04(66.7)$ & Nil & Nil & 06 \\
\hline
\end{tabular}

As depicted in table 3, P. aeruginosa and A. baumanii strains were most sensitive to Meropenem. S.maltophilia showed maximum sensitivity to ticarcillin/clavulanic acid whereas B. cepacia complex was found to be most sensitive to Piperacillin/Tazobactam, Meropenem and Ticarcillin/ Clavulanic acid.

Table 3: Antibiotic sensitivity pattern of nonfermenters

\begin{tabular}{lcccccccccc}
\hline \multicolumn{1}{c}{$\begin{array}{c}\text { Organism } \\
\text { (No. of strains) }\end{array}$} & $\mathbf{1}$ & $\mathbf{2}$ & $\mathbf{3}$ & $\mathbf{4}$ & $\mathbf{5}$ & $\mathbf{6}$ & $\mathbf{7}$ & $\mathbf{8}$ & $\mathbf{9}$ & $\mathbf{1 0}$ \\
\hline P. aeruginosa & 59 & 39 & 63 & 72 & 61 & 61 & 67 & 72 & 80 & 67 \\
A. baumannii & 42 & 13 & 19 & 13 & 16 & 26 & 35 & 52 & 81 & 39 \\
S.maltophilia & 31 & 15 & 38 & 62 & 15 & 15 & 31 & 38 & 62 & 77 \\
B. cepacia complex & 00 & 25 & 00 & 00 & 00 & 25 & 50 & 00 & 50 & 50 \\
\hline
\end{tabular}

1-Amikacin, 2-Aztreonam, 3-Ceftazidime, 4-Ciprofloxacin, 5-Gentamycin, 6- Piperacillin, 7- Piperacillin/Tazobactam, 8-Tobramycin, 9-Meropenem, 10- Ticarcillin/Clavulanic acid

\section{Discussion}

HAI is a worldwide phenomenon. In spite of awareness and hospital care, infections continue to develop in hospitalized patients and among hospital staff too. Factors promoting infections among hospitalized patients include decreased immunity; the increasing variety of medical procedures and invasive techniques creating potential routes of infection; and the transmission of drug resistant bacteria among crowded hospital populations, where poor infection control practices may facilitate transmission.

Over the last decade, NFGNBs have emerged as important opportunistic pathogens in the increasing population of patients who are immunocompromised by their disease or medical/surgical treatments. During routine clinical microbiology work in most laboratories, these are generally not pursued for identification due to the general notion of physicians that NFGNBs other than Pseudomonas aeruginosa are generally commensals or non pathogens and if required, can be treated easily with any of the broad spectrum antibiotics and also due to the tedious and time taking process of their species identification. ${ }^{8}$

In our study a total of $25.7 \%(94 / 519)$ NFs were isolated. It is higher than the studies done in Saudi Arabia ${ }^{9}$ and Orissa ${ }^{10}$ in which an incidence of $16 \%$ and $15.75 \%$ were reported, respectively. It was lower than that reported by the study done by KK Lahiri et $\mathrm{al}^{11}$ who had reported $31.2 \%$ from a tertiary care hospital. These differences in the prevalence of various bacterial isolates in different health care settings are likely and well expected as they depend on many local variables.

The demographic data in our study showed that the age group most commonly affected by NFGNB infections was that of more than 60 years which is in concordance with the findings of the study done elsewhere. ${ }^{12}$ The higher age group was more affected as these HAIs have a predilection for severely ill and immune compromised people.

The sex distribution is comparable and is in concordance with a study done by Rahbar et al ${ }^{12}$. This may be attributed to the fact that health care -associated infections do not have a discernible sex predilection.

It is a well-known fact that nosocomial infection rates in intensive care units (ICUs) are many times higher than elsewhere in hospitals as ICUs are the hub of severely ill patients, who are most prone to opportunistic infections. In present study too, highest incidence $(55.3 \%)$ of infections with NFGNB was reported from ICUs. Other studies have also reported higher incidences from ICUs. ${ }^{9,12}$

Tracking nosocomial infections by site has become difficult in the last few years because of shorter inpatient stays. For example, the average postoperative stay, now approximately 5 days, is usually shorter than the incubation period for many infectious diseases. There are many cases that are usually missed because of this reason. In this study, incidence of infections with NFs was found to be highest from respiratory specimens. This was similar to some studies ${ }^{13}$ but different to others ${ }^{14}$ in which pus samples were the most common specimen.

Nosocomial infections with Pseudomonas are quite frequent despite the application of various preventive measures in ICUs leading to increased morbidity, mortality, prolonged hospital stay and increased hospital expenses. In our study too, Pseudomonas aeruginosa was the most common non fermenter, accounting for $48.9 \%$ of the isolates. This was in consensus with some studies ${ }^{9}$, that have reported the incidence $42.41 \%$ and $56.0 \%$ respectively. Other studies ${ }^{4,15}$ from various parts of India have reported higher prevalence (more than $70 \%$ ) and $\mathrm{few}^{13}$ have reported lower prevalence 
i.e. less than $36 \%$, though in all the mentioned studies, Pseudomonas was the most common NF.

Ability of Acinetobacter bacteria to survive in hospital milieu and its ability to persist for extended periods of time on surfaces makes it a frequent cause of HAI. One of the most striking features of genus Acinetobacter is the ability to extremely rapid development of antibiotic resistance in response to challenge with new antibiotics. In India, very few studies on Acinetobacter species have been reported and in view of their increasing importance in nosocomial infections, further studies are warranted in this part of world. In our study prevalence of Acinetobacter baumannii was $33 \%$, which was comparable to many studies $9,11,12,15$ in which the prevalence was reported to be in the range of $28 \%$ to $36 \%$.

Resistance to most anti-Pseudomonal agents has increased by over $20 \%$ over the last five years. ${ }^{16} \mathrm{P}$. aeruginosa possesses more tools for defying the activity of any antimicrobial agents than virtually any other organism. ${ }^{17}$ Like Pseudomonas, Acinetobacter has been found to express a variety of different mechanisms including production of aminoglycoside modifying enzymes, ESBLs, carbapenemase, topoisomerases as well as through outer membrane proteins as penicillin binding proteins. ${ }^{18}$ Meropenem was the drug with highest sensitivity against Pseudomonas and Acinetobacter both in the present study, which was supported by other studies. ${ }^{12,19}$ Both of them were found to be most resistant against aztreonam.

In recent years, a significant increase in the incidence of Stenotrophomonas maltophilia has occurred, particularly in immune compromised individuals. Often, recovery of $S$. maltophiliat ends to be associated with colonization rather than true clinical infection, yet patients colonized with S. maltophilia are treated with antibiotics over extended periods, creating an opportunity for accelerated resistance development. In our study, $13.8 \%$ of isolates of S. maltophilia were isolated among all NFs which were comparable to the study done by Tang Bing et al ${ }^{20}$, who had reported the incidence to be $11.8 \%$. It was found to be most sensitive with Ticarcillin- Clavulanic acid (76.9\%) followed by Meropenem (61.5\%) and Ciprofloxacin (61.5\%) while against aztreonam, gentamicin and piperacillin, they were found to be least sensitive (15.4\%).

B. cepacia has the potential to cause fatal infections in the ICUs and its multidrug resistance makes its presence dangerous in hospital settings. It is intrinsically resistant to aminoglycosides and first- and second-generation cephalosporins. This resistance has been attributed to an impermeable selective outer membrane, an efflux pump mechanism, and/or the production of an inducible chromosomal beta-lactamase. ${ }^{21,22}$ That's why surveillance of $B$. cepacia infections should not be neglected, especially in the ICUs and due to its high transmissibility and previous epidemic reports, strict infection control measures should be applied in the case of a $B$. cepacia infection diagnosis. ${ }^{23}$ In our study $4.3 \%$ of non fermenters isolates were Burkholderia cepacia; $50 \%$ of them were sensitive to Ticarcillin/ Clavulanic acid, meropenem and piperacillin/ tazobactam while against most of the antibiotics they have shown complete resistance.

In our study 11 pan drug resistant strains were isolated out of which maximum number were Acinetobacter baumannii (5), followed by S. maltophilia (3), B. cepacia (2) and $P$. aeruginosa (1) and 21 XDR (extremely drug resistant) strains were isolated in our study. Among them 13 were Acinetobacter baumannii, P. aeruginosa (4), S. maltophilia (3) and B. cepacia (1).

\section{Conclusion}

Isolation of non-fermenters and their antibiotic susceptibility pattern should be regarded with all seriousness in clinical practice and clinical epidemiology because by being resistant to multiple antibiotics, their prevalence not only limits the treatment options but also act as a reservoir of drug resistance genes. They are emerging nosocomial pathogens and every effort should be made for prevention and control of infections caused by them which depends on practices of effective hospital infection control measures and minimization of risk factors.

Though in our study they showed good sensitivity against meropenem, Ticarcillin- clavulanic acid and piperacillinTazobactem, still development of resistance to even these drugs need to be closely monitored and analyzed further. Every hospital should identify these organisms on routine basis and based on the sensitivity pattern, it should have a laid down antibiotic policy for various clinical conditions. All treating doctors should adhere to the antibiotic policy and the policy should be revised regularly depending upon the antibiotic sensitivity pattern and feedback received from clinicians.

\section{References}

1. Benachinmardi KK, Padmavathy M, Malini J, et al. Prevalence of non-fermenting Gram-negative bacilli and their in vitro susceptibility pattern at a tertiary care teaching hospital. Indian J Med Microbiol 2014;41:162-6.

2. Livermore DM. Introduction: the challenge of multiresistance. Int J Antimicrob Agents. 2007;29 Suppl 3:S1-7. 
3. Yashodara P, Shyamala S. Identification and characterization of nonfermenters from clinical specimens. Indian J Med Microbiol 1977;15:195-7.

4. Vijaya D, Kamala, Bavani $S$ et al. Prevalence of nonfermenters in clinical specimens. Indian J Med Sci 2000;54:87-91.

5. Garner JS, Jarvis WR, Emori TG, et al. CDC definitions for nosocomial infections. Am $\mathrm{J}$ Infect Control 1988; 16:128-40.

6. Washington W, Jr. Stephen A, William J, et al. Koneman's Colour Atlas and Text Book of Diagnostic Microbiology 2006. 6th edition.

7. Clinical and Laboratory Standards Institute (CLSI formerly NCCLS). 2012. Performance Standards for Antimicrobial Disk susceptibility Tests; Approved Standard - Tenth Edition. M02-A11. CLSI, Wayne, PA.

8. Mayon-White RT, Ducel G, Kereseselidze $\mathrm{T}$ et al. An international survey of the prevalence of hospitalacquired infection. J Hosp Infect 1988;11(Supplement A):43-8.

9. Eltahawy AT, Khalaf RM. Antibiotic resistance among Gram-negative non-fermentative bacteria at a teaching hospital in Saudi Arabia. J Chemother 2001;13:260-4.

10. Mahapatra A, Samal B, Pattnaik D et al. Antimicrobial susceptibility pattern of clinical isolates of nonfermentative bacteria. Indian $J$ Path Microbiol 2003;46:526-7.

11. Lahiri KK, Mani NS, Purai SS. Acinetobacter spp as Nosocomial Pathogen: Clinical Significance and Antimicrobial Sensitivity. Med J Armed Forces India 2004;60:7-10.

12. Rahbar M, Mehragan H, Akbari NH et al. Prevalence of antibiotic-resistant Acinetobacter baumannii in a 1000-bed tertiary care hospital in Tehran, Iran. Indian J Pathol \& Microbiol 2010;53:290-3.

13. Lu Xi-chun, Zheng-xiang LIU, Xiao-ling LI, et al. Resistance analysis of Non fermentative Gram-negative Bacilli. Chinese J Nosocomiology 2009-11.

14. Kharangate NV, Pinto MJ, Rodrigues S, et al. Characterization of nonfermenters from clinical samples
Department of Microbiology, Goa Medical College, Bambolim. J Assoc Physicians India 2001;49:324-6.

15. Abhijit C, Jyotsna G, Gopal N. Characterization and antimicrobial susceptibility of clinical isolates of nonfermentative bacteria. Indian J Med Microbiol 2001;19;48-9.

16. Jung R, Fish DN, Obritsch MD, et al. Surveillance of multi-drug resistant Pseudomonas aeruginosa in an urban tertiary-care teaching hospital. J Hosp Infect 2004;57:105-11.

17. Rise LB. Challenges in identifying new antimicrobial agents effective for treating infections with Acinetobacter baumanii and Pseudomonas aeruginosa. Clin Infect dis 2006;43:100-5.

18. Gales AC, Jones RN, Foward KR, et al. Emerging importance of multidrug resistant Acinetobacter species and Stenotrophomonas maltophilia as pathogens in seriously ill patients, geographic patterns, epidemiological features and trends in the SENTRY antimicrobial surviellance program. Clin Infect dis 1977-79;32:104-13.

19. Meharwal SK, Taneja N, Sharma SK, et al. Complicated nosocomial UTI caused by nonfermenters. J Lab Physicians 2002; 18: 123-8.

20. Tang B, Ying-peng C, Zhu B, et al. Clinical Distribution and Drug Resistance of Non-fermentative Bacilli in Surgery. Chinese J nosocomiology 2004-07.

21. Burns JL, Wadsworth CD, Barry JJ, et al. Nucleotide sequence analysis of a gene from Burkholderia (Pseudomonas) cepacia encoding an outer membrane lipoprotein involved in multiple antibiotic resistance. Antimicrob Agents Chemother 1996;40:307-13.

22. Trépanier S, Prince A, Huletsky A. Characterization of the penA and penR genes of Burkholderia cepacia 249 which encode the chromosomal class A penicillinase and its LysR-type transcriptional regulator. Antimicrob Agents Chemother 1997;41:2399-405.

23. Murat D, Tunccan OG, Sezer BE et al. Nosocomial Burkholderia cepacia infections in a Turkish university hospital: a five-year surveillance. J Infect Dev Ctries 2009;3:273-7. 